\title{
Using lymphoscintigraphy as a prognostic tool in patients with cancer
}

This article was published in the following Dove Press journal:

Research and Reports in Nuclear Medicine

15 April 2016

Number of times this article has been viewed

\author{
Sergi Vidal-Sicart' \\ Renato A Valdés Olmos ${ }^{2}$ \\ 'Nuclear Medicine Department, \\ Hospital Clínic Barcelona, Barcelona, \\ Spain; ${ }^{2}$ Nuclear Medicine Section and \\ Interventional Molecular Imaging \\ Laboratory, Department of Radiology, \\ Leiden University Medical Centre, \\ Leiden, the Netherlands
}

\begin{abstract}
Early detection of metastatic involvement of lymph nodes is one of the greatest difficulties faced by the physicians. In the last few centuries, the lymphatic system has become an important field of interest in oncology. The lymphatic system functions in two opposing ways in cancer: defense against circulating tumor cells, and, on the other hand, a route for metastasis and a site of tumor growth when the defense mechanism fails. The significant impact of lymphatic dissemination on the staging, treatment, and outcome of solid cancers has stimulated investigations aimed at gaining more insight into several aspects of the lymphatic system. Its application in oncology has evolved in the last 50 years fostered by the recognition of the relationship of lymph node metastases to prognosis in several types of malignant tumors. Lymphatic mapping also offers the opportunity for repetitive studies undertaken for evaluation of the patient during follow-up, thereby offering early evidence of recurrence of the disease. More recent and widespread applications of lymphoscintigraphy form the basis for biopsy of the sentinel lymph node in patients with solid cancers. This article reviews the applications of lymphoscintigraphy in the current diverse oncologic clinical indications for the assessment of secondary lymphedema related to oncologic treatments and sentinel lymph node lymphatic mapping.
\end{abstract}

Keywords: lymphatic mapping, lymphedema, sentinel node, cancer

\section{Introduction}

Early detection of metastatic involvement of lymph nodes is one of the greatest difficulties faced by the physicians. The lymphatic system comprises a complex network of ducts and nodes distributed throughout the human body. It exhibits a remarkable variation similar to other anatomical structures such as the arterial or venous system. However, in contrast to the blood circulation, lymphatic flow is unidirectional. Lymph is similar to blood plasma and contains immune cells as part of the defense against microorganisms. Moreover, the excess of interstitial fluid returns to the blood circulation via the lymphatic system.

In the last few centuries, the lymphatic system has become an important field of interest in oncology. The lymphatic system functions in two opposing ways in cancer: defense against circulating tumor cells, and, on the other hand, a route for metastasis and a site of tumor growth when the defense mechanism fails. The significant impact of lymphatic dissemination on the staging, treatment, and outcome of solid cancers has stimulated investigations aimed at gaining more insight into several aspects of the lymphatic system.

Lymphoscintigraphy, that is, image mapping of lymph node basins and flow, represents one of the oldest applications of the radioactive tracer method. As early as in 
1953, Sherman and Ter-Pogossian described the nodal uptake of radiolabeled gold, following interstitial administration. ${ }^{1}$

This nuclear medicine imaging procedure provides information about the functional status of this system (lymph vessels and lymph nodes). It was originally introduced in clinical practice for identification of the causes of peripheral edema or for characterization of patients with lymph effusions. Thus, lymphoscintigraphy has been proved to be accurate for showing disturbances of the lymphatic system, mainly in the limbs., ${ }^{2,3}$

This technique reveals the major lymph vessels and demonstrates the absence or delayed lymph flow and reflux zones (backflow). Lymphoscintigraphy can reveal abnormalities of lymph uptake in nodes and can predict therapy response. ${ }^{4}$

On the other hand, its application in oncology has evolved in the last 50 years stimulated by the recognition of the relationship of lymph node metastases to prognosis in several types of malignant tumors. Thus, nodal disease as secondary to a malignant tumor is not only a prognostic marker, because it signals disease extension, but it could also act as a reservoir of malignant cells, which might, in turn, be a source of visceral metastases. ${ }^{5}$

For example, in those scenarios where therapy can be aggressive, lymphoscintigraphy provides the possibility of identifying some alterations in lymphatic nodes due to metastatic involvement that would have a remarkable impact on the clinical outcome. The identification of these alterations allows introducing more aggressive treatment schedules, which may result in a better survival. One classical example has been the utilization of this test to assess the inner mammary chain nodes as a prognostic factor in breast cancer. ${ }^{6}$

Lymphatic mapping can be repeated during the patient's follow-up to search for early proof of recurrence. More recent and widespread applications of lymphoscintigraphy form the basis for radioguided biopsy of the sentinel lymph node (SLN) in patients with solid cancers. This article reviews the applications of lymphoscintigraphy in the current diverse oncologic clinical indications for the assessment of secondary lymphedema related to oncologic treatments and SLN lymphatic mapping. ${ }^{7}$

\section{Lymphedema}

Lymphedema is a disease of the lymphatic system, where fluid accumulates in the interstitial space, resulting in swelling, adipose tissue hypertrophy, and fibrosis. ${ }^{8}$

This entity is classified into primary or secondary lymphedema. Primary lymphedema is provoked by a congenital alteration (anatomical or functional) in the lymphatic system.
Secondary lymphedema, the most frequent one, is caused by trauma, infectious disorders (mainly parasitic), surgery, or radiation in lymphatic vessels and nodes. One of the most frequent lymphedema appearances can be seen in patients with breast cancer following lymphadenectomy and/or radiotherapy treatment. ${ }^{9-11}$

Damage to the lymph-conducting pathways may occur secondary to any number of causes originating primarily outside the lymphatic system. In chronic venous insufficiency, as in postthrombotic syndrome, most of the interstitial fluid cannot return to the heart because of the obstructed veins. As a result, the volume in lower extremity lymphatics increases in order to compensate for the venous occlusion. This function of the lymph vessels continues until the lymphatic valvular mechanism becomes insufficient; then reflux appears, swelling of the limb increases, and ulcers develop. However, parasitic lymphedema is the most common cause of lymphedema worldwide. On the other hand, lymphedema frequently coexists with lipedema (bilateral and symmetrical inflammation of the lower extremities).

Secondary lower limb lymphedema is a frequent drawback of regional lymphadenectomy procedures or radiation therapy in gynecological malignancies. In these patients, lymphedema is chronic and, with some different severity grades, may result in mobility alterations, joint contractures, sensation disturbance, infection, and unpleasant appearance. ${ }^{12,13}$

The most common cancers in which treatment gives rise to lower limb lymphedema are melanoma, sarcoma, and pelvic tumors (including those of the cervix, uterus, and prostate); it is remarkable that cancer developed in pelvic cavity and sarcomas can present lymphedema. Disruption of the lymphatic channels (either from elective surgery or by accident) must be important to develop lymphedema due to the highly efficient regenerative potential of lymphatics. It is, probably, the failure of lymphatics to regenerate and reanastomose within fibrotic and altered tissue from irradiation that is responsible for lymphedema secondary to cancer treatment.

Upper arm lymphedema can be expected following axillary clearance and/or radiation therapy for breast cancer. The lymphedema incidence related to breast cancer ranges from $6 \%$ to $30 \%$ and increases with additional radiation therapy. Lymphedema is generally localized in the ipsilateral arm of the breast surgery, and this complication seems to be higher in those patients with elevated peripheral blood vascular filtration rate. ${ }^{14,15}$

A relationship between the number of lymph nodes harvested and the presence of lymphedema has not been clearly 
set yet, and current studies are focusing on diminishing the number of lymphadenectomies even when metastatic involvement of a lymphatic node is present. ${ }^{16}$

Therefore, early diagnosis and treatment to avoid progression of the illness and its side effects are necessary. Lymphoscintigraphy is an imaging modality used to diagnose the presence of limb lymphedema and characterize its severity as well as for the assessment of post-therapeutic results (Figure 1). ${ }^{17,18}$

Lymphoscintigraphy can be useful to predict response to therapy in patients with early stage extremity lymphedema. Quantitative lymphoscintigraphy is used to evaluate the seriousness of lymphatic impairment in upper extremity lymphedema after surgery as well as the therapeutic result in patients with leg lymphedema. ${ }^{19,20}$

Some studies demonstrated that the presence and gravity of dermal backflow on the lymphoscintigraphy are a prognostic indicator of bad response to therapy (Figure 1C). ${ }^{18,21}$

\section{Methodology for assessing lymphedema with lymphoscintigraphy}

Injection of radiotracers with subsequent lymphoscintigraphy has been a pivotal study for the assessment of
Table I Applications of lymphoscintigraphy

Differential diagnosis: Differentiation of the lymphatic system from vascular edema, myxedema, and lipedema

Evaluation: Assessment of lymphatic channels and lymphatic mapping Identification: Location and depiction of sentinel nodes from various malignancies

Quantitation: Lymph flow quantitation

lymphatic system since the 1950 s. This technique requires intradermal or subcutaneous injection of the radiotracer. Table 1 summarizes the clinical applications of lymphoscintigraphy.

Currently, ${ }^{99 \mathrm{~m}}$ Tc-labeled tracers such as antimony sulfide colloid, sulfur colloid, albumin colloid, and human serum albumin are the most used agents, and their use depends on the availability in each country. ${ }^{22}$

The large particle size of unfiltered ${ }^{99 \mathrm{~m}} \mathrm{Tc}$-sulfur colloid implies a low rate of absorption and slow progression to the nodes, requiring prolonged times for imaging. Thus, ${ }^{99 \mathrm{~m}} \mathrm{Tc}-$ filtered sulfur colloid (particle size $<100 \mathrm{~nm}$ ), commonly used in the USA for lymphoscintigraphy, is inexpensive, has a satisfactory safety profile, but also has its own disadvantages $(<5 \%$ is absorbed from the injection site and its progression from the area of subcutaneous administration is slow). To

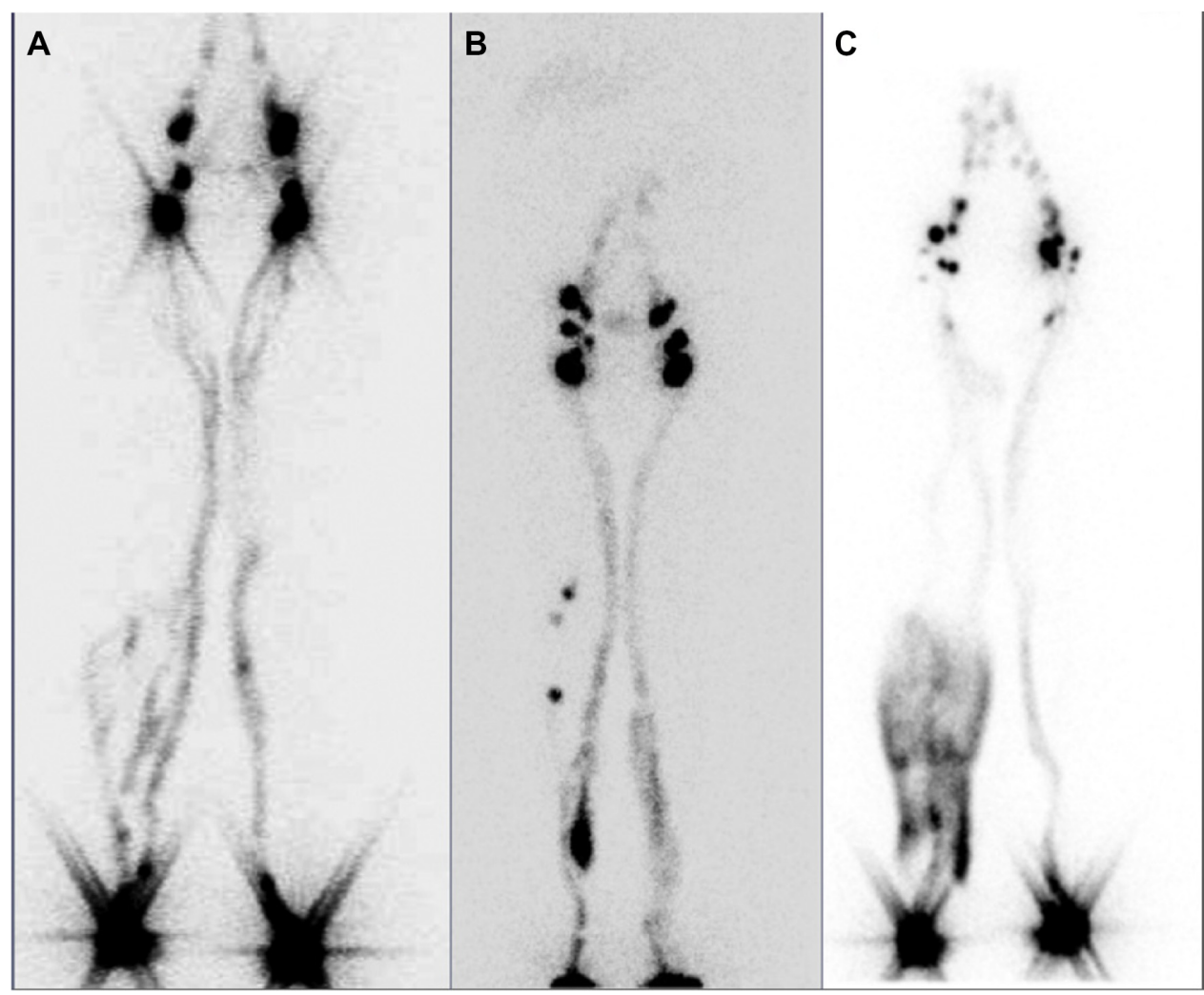

Figure I Lower limb lymphoscintigraphy.

Notes: The images show different lymphedema stages. Collateral circulation (A), lymphatic leakage (B), and dermal backflow (C). 
avoid this problem, filtering sulfur colloid using $0.1 \mu \mathrm{m}$ filter yielded a stable particle size of $<50 \mathrm{~nm}$ with great similarity with the antimony trisulfide colloid used in Australia and other countries. Albumin nanocolloid, mostly used in Europe, has a reasonable high homogeneous colloid size distribution $(95 \%$ is $<80 \mathrm{~nm}$ ), and it is easy to label with ${ }^{99 \mathrm{~m}} \mathrm{Tc}^{23}$

Subcutaneous and intradermal injections are commonly performed in studies of superficial lymphatics of the extremities. Mostbeck and Partsch compared both injections of ${ }^{99 \mathrm{~m}} \mathrm{Tc}-$ albumin nanocolloid and found that subcutaneous injections obtained more reliable results. ${ }^{24,25}$

The administration of noncolloidal agents ( ${ }^{99 \mathrm{~m}} \mathrm{Tc}$-human serum albumin) by intradermal injection shows a quick lymphatic transport, with a rapid assessment and better quantification of lymphatic flow.

The use of this approach (intradermal injection) in patients with primary lymphedema of lower extremity demonstrated a slow uptake. On the contrary, the uptake in lymphatic nodes was nearly normal in those patients with secondary lymphedema (although the use of subcutaneous injections suggested lymphatic obstruction). Therefore, there is still some controversy about which approach is best, although it has been reported that the subcutaneous way of injection is preferred for the colloidal agents. ${ }^{26}$

Injection of radiotracers in the subfascial area is frequently employed for the assessment of the deep system in the limbs. This injection is performed in the soles or in palms.

The bicompartimental lymphoscintigraphy (subcutaneous + subfascial) may be preferable for differentiation of the mechanisms of limb edema. A $4 \times 7-9 \mathrm{MBq}$ injection with $<1 \mathrm{~mL}$ of volume each is split into the space between the digits of the hands or the feet. Both extremities are injected because one of them serves as control for that with lymphedema. ${ }^{27}$

A whole-body imaging is recommended. A flood source is generally used for the depiction of body contour. The results are acquired at 10 minutes of injection and at 1-2 hours and 4-6 hours after the injection.

\section{Visual versus quantitative assessment}

Interpretation of the lymphoscintigraphic images may be performed by visual assessment or by means of a quantitative approach. In general, lymphoscintigraphy is a simple technique with flow patterns of normality, which are easily interpretable (symmetry of radiotracer migration, early visualization of lymph nodes, etc) by visual assessment of the results.

Lymphoscintigraphic visual interpretation includes a thorough evaluation of the injection site (delay and the presence or absence of lymphatic flow), lymphatic channels (symmetry versus asymmetry visualization), collateral vessels, dermal backflow, lymph nodes in lymphatic basins (number, size, and uptake of radiotracer) or the presence of lymph nodes in the deep lymphatic system (ie, popliteal and elbow nodes), and altered radiotracer accumulation due to extravasation, lymphocele, or lymphangiectasia. ${ }^{28}$

In abnormal lymphatic flow and lymphedema, different patterns may be observed: the presence of dermic backflow, asymmetry alteration in the groin or axillary lymph nodes, evidence of collateral pathways, visualization of in-transit lymph nodes (in ankle, popliteal fossa, or in the elbow), and the absence of tracer migration. The patterns of abnormality vary and do not seem to present the same diagnostic value. ${ }^{19,29}$

Lymphoscintigraphic visual assessment has been reported to have a high diagnostic performance, with a minimally invasive technique and discomfort. Absence of tracer migration from the injection site, presence of dermic backflow, and asymmetry of the inguinal/axillary lymph nodes were described to be the issues with the greater diagnostic precision of this technique. ${ }^{19,21,30}$ On the other hand, lymphoscintigraphic study can be performed after a "stress" intervention with the aim to increase the lymphatic flow (eg, changes in temperature, physical effort, or a given pharmacologic product). However, this approach is not generally used. In the lower extremities, stress actions include walking, standing, massage, treadmill, or cycling. In the arms, pressing and squeezing of a ball or massage are employed. ${ }^{18}$

After this selected activity (lasting 20 minutes), the patient is imaged again. When the lymph node appearance or the tissue clearance of the tracer change is observed, it means that a response to the stress intervention has been obtained. The lymphatic flow may be quantified, and it is very sensitive to the diagnosis of lymphatic alterations, although a quantitation of regional lymph node cumulative uptake is preferred. ${ }^{24,31,32}$

The quantitative studies take into consideration drawing regions of interest on injection sites and axillary/inguinal nodes to quantify the tracer accumulation on these nodes and the radiopharmaceutical clearance from the injection site.

Regions of interest are performed on the early and delayed images, and a comparison between them is obtained with an equation for the radiotracer clearance and nodal uptake.

In general, studies reported no differences in radiotracer clearance, and the discrimination between healthy patients and patients with lymphedema using the clearance from the injection site was not possible. On the other hand, regional 
smaller lymph node uptake of radiotracer was present mainly in the strongly altered lymphatic flow.

Some reports demonstrated that this quantitative analysis improves the sensitivity and specificity of lymphoscintigraphy in diagnosing lymphedema. ${ }^{33,34}$

Weissleder and Weissleder studied 308 lymphedema extremities in a qualitative and quantitative way. Their results demonstrated that qualitative analysis confirmed lymphedema in $70 \%$ of cases. However, quantitative approach demonstrated functional lymphatic abnormalities in all cases. Qualitative analysis missed mild, grade I limb lymphedema in $30 \%$ of extremities. ${ }^{35}$ The data obtained suggest that the quantitative method may be a more sensitive approach to differentiate normal patients or those with mild lymphedema.

However, the study results including a high number of subjects consider that neither the lymphoscintigraphic pattern nor the use of quantitative studies is able to differentiate between primary and secondary lymphedema. Although quantitative methods of evaluation may be a useful tool, qualitative analysis of the images shows a high sensitivity and specificity. ${ }^{36}$

\section{Prognostic value of lymphoscintigraphy for lymphedema assessment}

Szuba et al found, in women undergoing therapy for postmastectomy lymphedema, that the state of the lymphatic function before therapy correlated with the final result of manual therapy. ${ }^{19}$

Therefore, when a postsurgical lymphoscintigraphic study in patients with breast cancer who underwent lymphadenectomy depicts disturbances of lymphatic drainage, the risk of upper limb lymphedema increases. Women with axillary lymph node clearance and radiation therapy who present an impaired lymphoscintigraphy several months after therapy can be considered at risk of developing arm lymphedema. Therefore, postoperative lymphoscintigraphy is a useful tool to select those patients with an increased risk of developing limb lymphedema. The importance of a good and early diagnosis of this group of patients may result in the implementation of the best approach to decrease the risk of lymphedema. ${ }^{17,37}$

In this group of patients, the degree of lymphatic functional impairment prior to the therapy, as assessed by lymphoscintigraphy, correlates inversely with manual therapy results. $^{38}$

Similarly, in patients with stage I extremity lymphedema, lymphoscintigraphy can predict the long-term response to physical therapy. In this regard, the visualization of a main lymphatic vessel without collateral lymphatic vessels has been reported to be the best predictor for a favorable response, as it has been the case with persisting lymph node visualization 4 hours after radiocolloid administration. ${ }^{39,40}$

Lymphoscintigraphy may also be used to predict the risk of developing lymphedema. In fact, in patients who have been operated for breast cancer, abnormalities of lymphatic drainage in a lymphoscintigraphy obtained after surgery increase the risk of developing upper limb lymphedema. ${ }^{41}$

Moreover, functional lymphatic changes detected by lymphoscintigraphy after external beam radiation therapy can predict the development of upper limb lymphedema. Recently, lymphoscintigraphy, including single-photon emission computed tomography (SPECT)/computed tomography (CT) of the axillary region, has been employed to evaluate the impact of including, as target volumes in the radiation treatment plan, the lymph nodes involved in arm drainage that might affect lymphedema. ${ }^{42}$

\section{Lymphatic mapping and sentinel node approach}

Numerous tumors spread their metastases to regional lymph nodes, including melanoma, breast, oral, gastrointestinal, and gynecologic and urogenital cancers. Lymphatic involvement is the most important prognostic factor in many malignant tumors. Staging depends on this involvement, and it usually implies important decisions related to adjuvant treatment. Some imaging diagnostic methods (CT, magnetic resonance imaging, ultrasound, etc) have been applied to rule out nodal involvement, but, despite their use, they are not accurate enough to avoid invasive techniques. For example, lymphadenectomy has become a classical diagnostic tool in breast cancer staging. Regional lymph nodes were harvested and studied for pathologic processing of lymphadenectomy specimens to search for metastases. Until the 1990s, prophylactic lymphadenectomy was used for breast cancer and melanoma; this approach put a high number of patients at risk of complications related to this surgical intervention, including lymphedema and infection. Due to the associated morbidity and some major limitations, more conservative methods have been developed with the intention to avoid lymphadenectomy.

Cabanas initially coined the SLN concept in 1977 when he used lymphangiography to assess patients with penile cancer. $^{43}$

For many years, lymphoscintigraphy was used in patients with melanoma to guide surgeons to the most likely nodal basin that harbors metastasis, especially when the primary tumor 
was located in regions with highly variable drainage. In 1992, Morton et al introduced the current concept of SLN mapping and biopsy in a series of patients with melanoma. ${ }^{44}$

This seminal work described a minimally invasive technique that could be used for increasing the accuracy of cancers while diminishing the morbidity. The biopsy of the SLN is able to provide accurate prognostic information, demonstrate the necessity of performing a lymphadenectomy, and select those patients for adjuvant treatment. Melanoma and breast cancers were the first clinical scenarios for SLN biopsy, although other applications have been validated since then (oral cavity, urogenital, and gynecological cancers). ${ }^{45-47}$

The concept of SLN biopsy in oncologic surgery is related to the Halstedian theory that any solid tumor drains in an orderly fashion via the lymphatic system, from the lower to the upper lymphatic stations. Therefore, the SLN would be the first node to be involved by metastasis, while a negative SLN represents that it is unlikely that other nodes located in the same lymphatic pathway will be involved. It has further been assumed that if no tumor cells are observed in the SLN, the morbidity related to lymph node dissection could be avoided. This assumption has been confirmed in clinical trials carried out mostly in patients with breast cancer or melanoma, as well as in patients with penile, vulvar, or oral cancer. As a result, SLN evaluation has become an integral component of treatment planning in patients with these tumors, particularly for staging and prognostic purposes. ${ }^{48}$

Lymphoscintigraphy is accepted as an essential part of the SLN mapping. It demonstrates the passage of radioactive tracers through the normal lymphatic structures and displays any alternative route of flow to different lymphatic basins. Visualization of all lymph nodes located along the route of the lymph will occur, and other nodes or paths will be seen if flow has been redirected because, for example, a blockage of the normal tract. Thus, lymphoscintigraphy provides a visual roadmap of nodal locations before surgery and may depict the chronological order in which lymphatic flow reaches lymph nodes, especially when a dynamic study is performed. However, despite the experience acquired over so many decades, protocols for performing lymphoscintigraphy are not standardized yet, and remarkable differences between centers still persist. ${ }^{49-56}$

The radiotracer can move to more distally located nodes that those geographically closer to the primary tumor site, and this occurrence can only be seen by imaging. ${ }^{57}$

On the other hand, there are several issues in the clinical scenario that increase or decrease the lymphatic flow. When performing the lymphoscintigraphy, some of these factors (heat, cold, massage, and inflammation) may influence the accuracy of the lymphatic mapping. Moreover, we must keep in mind that the velocity of lymphatic flow is not the same in all regions of the body and varies depending on the area of the skin. ${ }^{58}$

Thus, the average flow in the head and neck region is $1.5 \mathrm{~cm} / \mathrm{min}$, increases to $3.9 \mathrm{~cm} / \mathrm{min}$ in the posterior trunk, and is higher in the forearm and hand with $5.5 \mathrm{~cm} / \mathrm{min}$. When the lymphatic flow goes faster, more second-echelon nodes beyond SLN are visualized. Second-echelon nodes are more frequently observed in the groin than in other parts of the body, due to the highest velocity of lymph seen from the lower limb (10.2 cm/min).

Lymphoscintigraphy for SLN mapping must be extremely accurate and reproducible. In this sense, some studies have addressed this issue in several different malignancies. Thus, in patients with melanoma, a good relationship (84\%-96\%) between two separated lymphoscintigraphic explorations has been demonstrated. ${ }^{59-64}$

Tanis et al elegantly demonstrated a $100 \%$ concordance between two lymphoscintigraphies in 25 patients with breast cancer. ${ }^{65}$ The same excellent reproducibility was achieved in penile cancer. ${ }^{6}{ }^{6}$ Lymphoscintigraphy predicts the coordinates of the SLN to the surgeon before dissection and provides more confidence in the subsequent handheld gamma probe localization. When tracer flows through lymphatic vessels or lymph nodes are delayed, some SLNs may not be detected with imaging (this circumstance depends on several clinical situations, injection procedures, and node status). However, some of them can be found during surgery by the gamma probe. ${ }^{67}$

Because the SLN concept is applied for different tumors, various imaging protocols are used. Lymphoscintigraphy imaging should start as soon as possible (especially in fast lymphatic flow superficial tumors, ie, melanoma and skin malignancies, oral cavity tumors, and vulvar and penile cancer) because the tracer can quickly reach the SLNs. Early images may display the lymphatic channels, which can be followed to the SLN and subsequently marked on the skin surface as a guide for surgery. The visualization of multiple lymph channels can identify several different SLNs (Figure 2). ${ }^{68-70}$

Lateral and oblique images may provide information about the depth of the SLN, but currently, the introduction of SPECT/CT has revolutionized the imaging scenario. The functional information provided by SPECT can be combined with the morphological information from CT in the same session. The final SPECT/CT fused images depict SLNs in an anatomical environment allowing a helpful map for surgeons. 


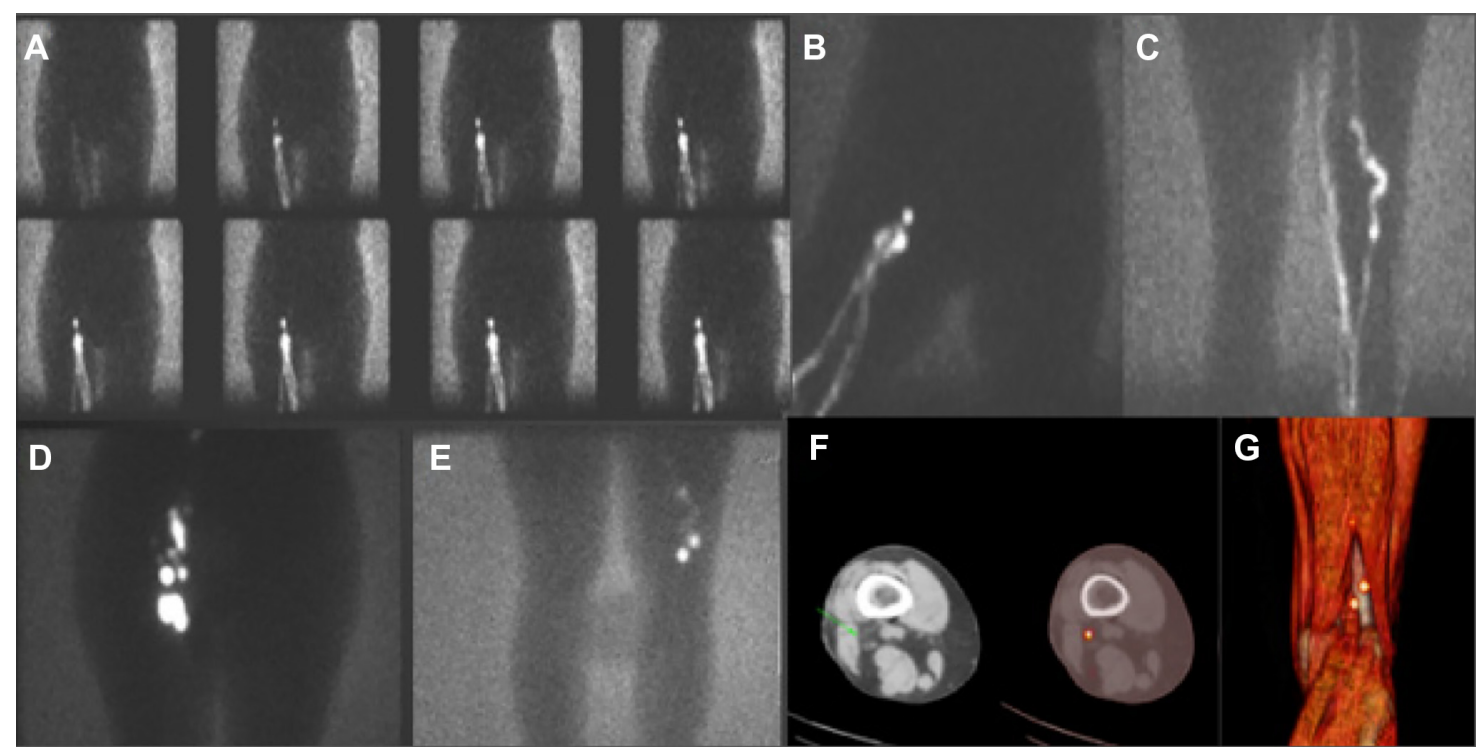

Figure 2 Patient with melanoma in right heel.

Notes: Importance of dynamic study for depicting lymphatic channels (A)-(C). Delayed images show inguinal nodes (D) and popliteal nodes (E). Axial fused slice of SPECT/CT showing the location of popliteal SLN (F). Popliteal 3D reconstruction (G).

Abbreviations: 3D, three dimensional; SLN, sentinel lymph node; SPECT/CT, single-photon emission computed tomography/computed tomography.

SPECT/CT has been used in patients with melanoma and breast cancer with infrequent or complex drainage. Furthermore, SPECT/CT is of great importance to accurately localize lymph nodes located in difficult-to-find areas such as the pelvis and abdomen in gastrointestinal, gynecological, and urological cancers (Figure 3). ${ }^{71-73}$

SPECT/CT does not replace planar lymphoscintigraphy. It must be considered a complementary modality because SPECT/CT may detect additional SLNs. In the current protocols, SPECT/CT is acquired after delayed planar images. This sequential acquisition is of great importance to clarify the role of both modalities. For imaging interpretation, it has been reported that the major criteria to identify lymph nodes as SLNs are the visualization of lymphatic ducts, the time of appearance, the lymph node basin, and the intensity of lymph node uptake. With this information in mind, visualized lymph nodes may be classified as follows:

1. Definitively SLNs: This category includes all lymph nodes draining from the site of the primary tumor through its own lymphatic vessel or a single radioactive lymph node in a lymph node basin.

2. Highly probable SLNs: This includes nodes appearing between the injection site and a first visualized node, or nodes with increasing uptake appearing in other lymph node stations.

3. Less probable SLNs: All higher echelon nodes may be included in this category.
Using these lymphoscintigraphic categories is helpful for surgical decision making. Lymph nodes reported to be included in the first two categories must be removed during surgery. Less probable SLNs may sometimes be removed depending on the degree of remaining radioactivity measured by the gamma probe or the portable gamma camera during the control of the excision. ${ }^{74}$

The surgical guidance, taking into account the information given by SPECT/CT, is achieved by conventional handheld gamma probes and more recently with portable gamma cameras. This collaboration between technologies allows to refine the approach and results of radioguided surgery. ${ }^{75,76}$

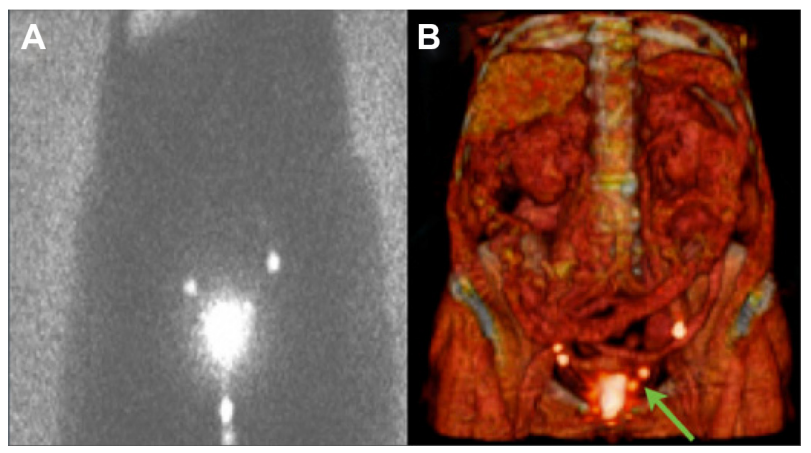

Figure 3 Endometrial cancer.

Notes: Planar anterior image showing bilateral pelvic drainage (A). SPECT/CT data and $3 \mathrm{D}$ reconstruction improves the visualization of close nodes in parametrium (green arrow) (B).

Abbreviations: SPECT/CT, single-photon emission computed tomography/computed tomography. 
The possibility to combine the radiotracers with other agents opens a new field to explore. In this sense, ${ }^{99 \mathrm{~m}} \mathrm{Tc}-$ nanocolloid has been combined with indocyanine green (ICG), a fluorescent agent for lymphatic mapping. This hybrid tracer permits the surgeons to integrate the standard approach based on radioguided detection with a portable gamma probe or camera with a new optical modality based on fluorescent signal detection. This methodology is being applied in several malignancies with a high degree of success. ${ }^{77-79}$ There are reports on active SLN-targeting agents, such as a multimodal imaging tracer combining ${ }^{68} \mathrm{Ga}$ and/or ${ }^{99 \mathrm{~m}} \mathrm{Tc}$ with a near-infrared dye IRDye $800 \mathrm{CW}$, a heptamethine cyanine dye that can be readily attached to biomarker-targeting molecules such as monoclonal antibodies and peptides, which enables positron emission tomography (PET)/CT and fluorescence imaging. ${ }^{80,81}$

Some merits of this tracer are as follows: the dextran backbone is not a serum-derived product and may have advantages in certain countries, where albumin is not in favor; the optical reporter is covalently attached to the backbone; and there is also an active targeting motif to the SLNs. This is unlike the nanocolloid case, where indocyanine green is absorbed via intermolecular forces. Radiolabeling with $68 \mathrm{Ga}$ facilitates tracking of the $800 \mathrm{CW}$ tilmanocept in vivo with a PET scanner. Additionally, the agent can be visualized by fluorescence and/or by a handheld radioactivity detector. This agent has shown some promises in preoperative planning by PET/CT and intraoperative guidance via fluorescence. ${ }^{82,83}$

\section{Prognostic implications of lymphoscintigraphy for SLN mapping Melanoma}

A great discordancy was observed between clinically predicted lymphatic drainage areas and the pathways depicted in lymphoscintigraphy. ${ }^{84}$ This is relevant, bearing in mind that the most important predictor of prognosis and recurrence for early melanoma is the status of the SLN. In one study, SLN biopsy was performed in 365 patients with head and neck melanoma. SLNs were located in $98.6 \%$ of cases $(3.7$ nodes removed per patient and an average of 1.6 nodal basins per patient). The SLN was metastatic in $40(11 \%)$ patients. SLN-positive patients presented thicker melanomas, higher recurrence, and a significant decrease in overall survival in comparison with patients who had negative SLNs. After a median follow-up of 8 years, 17 out of 365 SLN-negative patients developed regional recurrence for a false-negative rate of $5.2 \%$ and a negative predictive value of $94.8 \%{ }^{85}$
In another study concerning patients with head and neck melanoma, SLNs were identified in $94.7 \%$ of patients compared with $95.3 \%-100 \%$ detection rate in other anatomic areas. A higher number of false-negative cases were observed in those patients with primary melanoma in head and neck. A positive SLN was associated with both lower disease-free survival (53.4\% versus $83.2 \%$ ) and overall survival ( $40 \%$ versus $84 \%$ ). The conclusions of that study highlighted that SLN biopsy should be offered to all patients with intermediateand high-risk melanomas in the head and neck area. ${ }^{86}$

In a German multicentric study, 2,653 patients were included. The estimated negative predictive value of SLN approach was $96.4 \%$. The false-negative rates after 1 year, 2 years, 3 years, 5 years, and 10 years were estimated to be $2.5 \%, 4.6 \%, 6.4 \%, 8.7 \%$, and $12.6 \%$, respectively. Factors that increase the potential false-negative results were older age, fewer SLNs excised, and head or neck location of the melanoma. Compared with SLN-positive patients, the falsenegative cases presented lower survival. SLN-positive patients presented a nodal basin recurrence rate at 5 years of $18.3 \%$. The recurrence rates for axilla, groin, and neck were $17.2 \%$, $15.5 \%$, and $44.1 \%$, respectively. Local relapse after lymphadenectomy was higher with older age, head, or neck location of the melanoma, ulceration, central metastasis into the SLN, and $>2$ lymph node involvement. False-negative cases were associated with fewer SLNs excised. Thus, lymphoscintigraphy played a key role for ascertaining all potential SLNs. ${ }^{87}$

Some studies were undertaken to assess whether imaging from lymphoscintigraphy could predict a higher falsenegative rate if only the nodes with highest uptake were removed. In one of them, Ho et al assessed 67 patients. All lymph nodes with radioactivity $>10 \%$ of the most radioactive nodes were harvested. In 13 out of 67 (19\%) patients, the most radioactive lymph node was not involved, while a less radioactive node presented metastasis. Imaging by lymphoscintigraphy presented in this study is as follows: sensitivity $31 \%$, specificity $91 \%$, positive predictive value $44 \%$, and negative predictive value $85 \%$. Therefore, it seems that lymphoscintigraphy shows a high specificity and negative predictive value but lower sensitivity for detecting when the SLN will not be the most radioactive lymph node. These results foster the necessity to perform dynamic studies in the lymphoscintigraphic methodology, but the imaging protocol could certainly benefit from further optimization. ${ }^{88}$

Lymphoscintigraphy depicts second-echelon nodes in many patients and SLNs that do not present the highest uptake. Luo et al evaluated the clinical significance of melanoma in SLNs that were not SLN with highest uptake 
in order to determine the risk of disease progression. A total of 1,575 SLNs were analyzed in 475 patients. Ninety-one patients (19\%) had positive SLNs. Of these, 72 (79\%) presented metastases in the most active SLN. In 19 cases with involved nodes (that were not the hottest SLN), counts varied from $26 \%$ to $97 \%$ of the hottest node. In patients with negative SLNs, 11\% developed metastases beyond the lymph node basin and $3.4 \%$ recurred in the basin. Thus, removing only the most active SLN would be a false-negative node in 19 of 475 (4\%) of all patients and 19 of 91 patients $(21 \%)$ with positive SLNs. The authors emphasized the importance of removing the less hot nodes, as depicted in preoperative lymphoscintigraphy. ${ }^{89}$

The advent of SPECT/CT may improve these figures from the planar lymphoscintigraphy. ${ }^{90}$ Stoffels et al studied the lymphatic mapping in 254 patients (149 with SPECT/CT). In the SPECT/CT group, more SLNs per patient were depicted in comparison with the standard group (2.40 versus $1.87 ; P<0.001)$. Positive SLNs per patient was significantly higher in the SPECT/CT group than in the standard $(0.34$ versus $0.21 ; P=0.04)$. The local relapse rate in the SPECT/ $\mathrm{CT}$ cohort was lower than in the standard cohort $(6.8 \%$ versus $23.8 \% ; P=0.03)$, which prolonged 4-year disease-free survival $(93.9 \%$ versus $79.2 \% ; P=0.02)$. The authors stated that the use of SPECT/CT is of great value compared with SLN alone and it was associated with a higher number of metastasis detection and an increase in disease-free survival. ${ }^{91}$

Moreover, Veenstra et al previously demonstrated the importance of adding SPECT/CT to classical lymphoscintigraphy. They studied 35 patients with melanoma scheduled for wide local excision and SLN biopsy. All underwent conventional lymphoscintigraphy and SPECT/CT. The images provided by SPECT/CT showed the same 69 SLNs as planar images in all 35 patients. However, SPECT/CT depicted eight additional SLNs in seven patients (20\%). In two of these patients $(5.7 \%)$, SPECT/CT showed nodes in additional nodal basins that were subsequently explored to detect the extra SLNs. This additional information provided by SPECT/CT was helpful in eleven patients (31\%). ${ }^{92}$

\section{Breast cancer}

Lymphoscintigraphy was demonstrated to be of utmost importance for accurate and satisfactory SLN retrieval during surgery in the ALMANAC trial. The authors found that the success of SLN biopsy decreased when there was nonvisualization of lymphatic nodes on the preoperative lymphoscintigraphy. The SLN was located during surgery in $98 \%$ of patients who had SLNs previously visualized on lymphoscintigraphy. In patients without SLN visualization on previous lymphatic scan, the SLN identification rate during surgery decreased to $90 \%$. Moreover, intraoperative SLN identification using only the gamma probe was higher in the group with positive lymphoscintigraphy visualization than in the group without SLN depiction on preoperative images $(95 \%$ versus $68 \%) .{ }^{93}$

The axillary lymph node status is currently one of the most important prognostic factors in breast cancer. However, nodal involvement outside the axilla may be observed in up to $56 \%$ of these patients. ${ }^{94}$ Lymphoscintigraphy shows lymphatic drainage outside the axilla, especially to the inner mammary nodes (IMN), in $20 \%-30 \%$ of patients with breast cancer, when intratumoral or peritumoral tracer injections are used (Figure 4). ${ }^{95,96}$

With intraparenchymal injection technique, IMN drainage can be frequently observed, but subareolar injection, which improves axillary node detection, rarely show IMN uptake. ${ }^{97}$ Most of IMN metastases have axillary involvement at the same time, but up to $10 \%$ of patients may present IMN metastases exclusively. This issue is classified as stage $\mathrm{N} 3$, and it has been associated with a potential increase in distant metastases and, subsequently, a decrease in overall survival rates. ${ }^{98,99}$

Several studies found that breast cancers located in medial areas of the chest had a worse prognosis compared with breast cancers located in lateral areas. It was believed that this worse outcome could be related to untreated IMN metastases, which are more frequent in medial breast cancers. ${ }^{100,101}$

In studies, where biopsy was performed for IMN due to the presence of an SLN on lymphoscintigraphy, metastases were detected in $8 \%-27 \%$ of patients. However, a great majority of surgeons are reluctant to perform IMN SLN retrieval because of difficulties in the surgical approach and the lack of convincing data for survival benefit. ${ }^{101}$

Nevertheless, two studies showed that IMN retrieval improved breast cancer staging and a better 5-year survival after selected radiotherapy and systemic therapy. ${ }^{102,103}$

In several studies, including a total of 3,876 patients with SLN biopsy, parasternal drainage was demonstrated in 792 patients (20.4\%). Successful IMN SLN biopsy was performed in 644 patients, and a positive SLN was obtained in 111 cases (17.2\%). Of these positive SLNs, $40 \%$ had the primary cancer located in the lateral breast quadrants. Importantly, in patients without axillary involvement, the IMN SLN biopsy was positive in $7.8 \%$ of cases. On the contrary, in patients with metastatic SLN in axilla, the IMN SLN biopsy was positive in $41 \%(P<0.00001)$. Thus, patients with IMN drainage on 


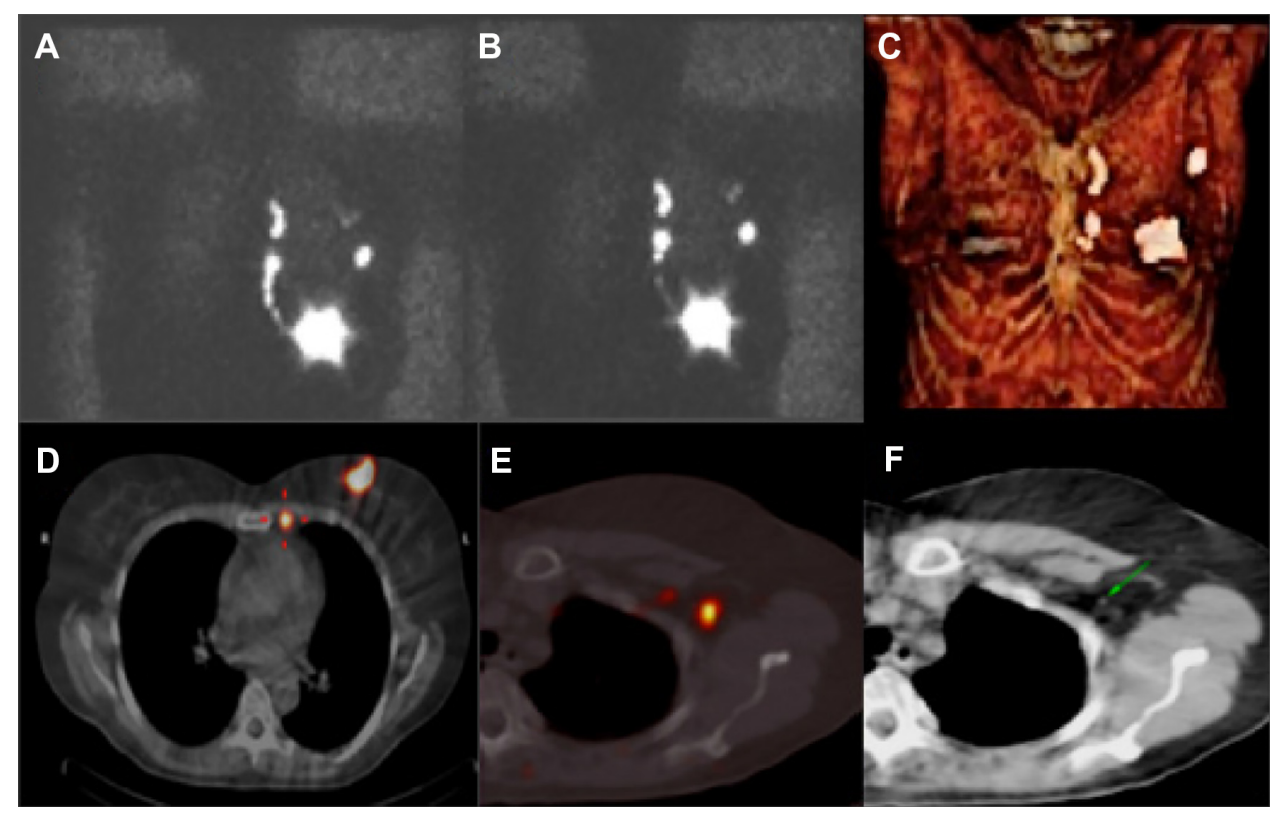

Figure 4 Patient with breast cancer with drainage to axillary and internal mammary chain.

Notes: Early (A) and delayed images (B). Volume rendering reconstruction (C). Axial slice showing the IMN SLN (D). Axial fused slice with axillary SLN (E) and the same node depicted on CT component $(\mathbf{F})$.

Abbreviations: CT, computed tomography; IMN, inner mammary node; SLN, sentinel lymph node.

lymphoscintigraphy and a positive axilla SLN have a high risk of occult IMN. These women should be considered for extended radiotherapy in the parasternal area. ${ }^{104}$

\section{Prostate cancer}

Lymphoscintigraphy and SPECT/CT are essential for predicting the lymphatic drainage from prostate cancer. In many cases, early-appearing lymph nodes seen as single on planar images are displayed as two or more separate lymph nodes in different basins by SPECT/CT, thereby increasing the number of nodes considered as SLNs. In other cases, intense lymph node uptake in a lymphatic basin seen on fused images may correspond to a cluster of SLNs as depicted on the CT of the SPECT/CT. Thus, SPECT/CT can depict more SLNs than planar images, and in one series, an average of 4.3 SLNs on SPECT/CT versus 2.2 SLNs on planar images was reported in 46 patients; $44 \%$ of the SLNs containing metastatic cells were visualized by SPECT/CT fused images only. ${ }^{105}$ Meinhardt et al found that lymphoscintigraphy and SPECT/CT identified SLNs outside the area of extended pelvic lymphadenectomy in $31 \%$ of cases. These SLNs were found in different locations: paraaortic, para-rectal, behind the common iliac artery, presacral, Cloquet's node, inguinal, lateral to the external iliac artery, and in the abdominal muscular wall. ${ }^{106}$ The SLN rate outside the lymphadenectomy area increases in patients previously treated with radiotherapy or surgery for prostate cancer to an $80 \%$ in comparison with a $34 \%$ for untreated patients. ${ }^{107,108}$

\section{Penile cancer}

In penile lymphoscintigraphy, the dorsal lymphatic channels of the penis are observed first, immediately after radiocolloid injection. The pattern of visualization most frequently observed ( $80 \%)$ is bilateral to both inguinal regions. This pattern is, however, asynchronous in nearly $66 \%$ of the patients, and late SLN uptake of the contralateral side is only visualized on delayed imaging. ${ }^{109}$ Drainage from the injection site occurs, in a majority of patients, through one or two lymphatic channels, leading to visualization of one or two SLNs in each groin. The main advantage of SPECT/CT is its possibility to distinguish inguinal from iliac nodes. The SLNs are generally located in Daseler's superior and central inguinal zones, which are cranial to and directly overlying the saphenofemoral junction, respectively. Higher tier lymph nodes were also located in these inguinal zones and in the pelvic region. These findings suggest that the extent of inguinal lymphadenectomy could be reduced to the removal of the superior and central inguinal zones. This surgical approach may decrease the postoperative morbidity associated with the procedure. ${ }^{110}$

\section{Vulvar cancer}

Similar to urologic malignancies, gynecologic tumors spread regionally to the pelvic lymph nodes with high frequency. Functional imaging techniques allow this potential lymphatic spread assessment, and they are often used for a better staging of gynecologic malignancies. ${ }^{111}$ 
Lymphoscintigraphy is helpful in vulvar cancer, especially when several SLNs are visualized. The correlation between SLNs observed on the lymphoscintigraphy and those excised during surgery showed good agreement in some studies and are not good enough in others. However, some nodes located on unexpected lymphatic areas can be missed due to the lack of a lymphatic map of the tumor. Using SPECT/CT additionally to lymphoscintigraphy, Collarino et al demonstrated that SLNs in $93 \%$ of the cases are located in the medial and central inguinal zones of Daseler. However, a 7\% lymphatic drainage is observed on the lateral areas of the groin, with SLNs in some cases located at a distance of various centimeters from the medial zones. ${ }^{72,112}$

Bilateral drainage is expected in vulvar tumors placed on midline or until $2 \mathrm{~cm}$ from the midline; this means that a lack of drainage to one of the two sides of the inguinofemoral regions requires further actions to ensure the bilateral drainage (repeat injection of the tracer or SPECT/CT). Lateralized vulvar tumors are considered an exception when located in the major labia, $2 \mathrm{~cm}$ away from the midline. In these cases, unilateral drainage is accepted, but if bilateral drainage is found, all SLNs must be removed, including those on the contralateral region (Figure 5). ${ }^{113,114}$

The results obtained in the GOG173 and GROINSS-V trials demonstrated that SLN approach must be offered to a selected group of patients with vulvar cancer (tumor size $<4 \mathrm{~cm}$ and clinically negative groins). ${ }^{115,116}$

However, one important question is a matter of concern: which patients would undergo unilateral SLN biopsy with a great degree of confidence? For some authors when vulvar tumors are located in midline zone, a complete inguinofemoral lymphadenectomy is mandatory in the groin where SLN is not observed. ${ }^{117,118}$

Coleman et al studied 234 patients with vulvar cancer who were included in the GOG173 trial. Lymphoscintigraphy showed bilateral drainage in $22 \%$ of tumors located in lateral area, $58 \%$ of tumors in areas close to midline, and $70 \%$ of midline tumors. No contralateral metastasis was observed in patients with close-to-midline tumors, and unilateral drainage was depicted only in four of $32(12.5 \%)$ patients with midline tumors. Therefore, SLN biopsy might be safely performed only in this side in patients with lateral, close-to-midline tumors, and ipsilateral drainage on lymphoscintigraphy. ${ }^{119}$

\section{Cervix cancer}

In cervical cancer, the SENTICOL study confirmed the suitability and utility of SLN biopsy in this malignancy (early stages). SLN detection was successful in $>95 \%$ of cases, with bilateral drainage and SLN retrieval in $76 \%$ of patients. The false-negative rate was $8.0 \%$ in the complete group of patients and $0 \%$ among those with bilateral drainage and SLN retrieval. The rate of bilateral detection was higher in younger patients. ${ }^{120}$

Importantly, a less number of SLNs were retrieved during surgery in those cases with unilateral drainage seen on preoperative lymphoscintigraphy. Therefore, the detection of fewer SLNs during surgery, or of SLNs at fewer sites, compared with lymphoscintigraphy might increase the falsenegative rate. The combination of lymphoscintigraphy and SPECT/CT may decrease this risk. Unilateral SLN detection, large tumor size $(>20 \mathrm{~mm})$, and location of node metastases in the parametrium are associated with a higher risk of false-negative results. In SENTICOL study, although the SLN detection rate was lower by lymphoscintigraphy than by surgical procedure, the remarkable number of SLNs visualized by lymphoscintigraphy in unusual pelvic or abdominal areas provided valuable guidance during surgery. ${ }^{121,122}$

In conclusion, if the SLN is not found during lymphoscintigraphy or surgery, standardized pelvic lymphadenectomy

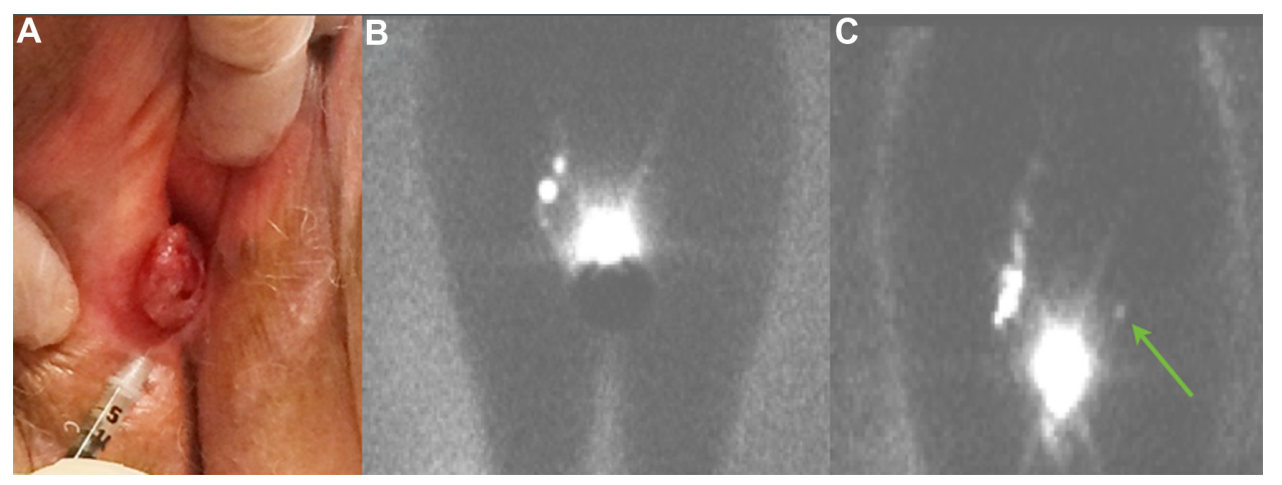

Figure 5 Vulvar cancer.

Notes: Central lesion (A). Unilateral right drainage in the early image (B). Bilateral drainage with a very faint SLN in the left groin in the delayed image (C). Abbreviation: SLN, sentinel lymph node. 
should be performed instead. Therefore, in cervical cancer, if the SLN is detected only on one hemipelvis, systematic lymphadenectomy should be performed on the other side. ${ }^{123,124}$

\section{Endometrial cancer}

In endometrial cancer, the normal pattern of lymphatic drainage includes pelvic and para-aortic lymph nodes. Visualization of para-aortic lymph nodes is very low in the case of cervical injection (only up to $11 \%$ of cases and without exclusive para-aortic SLNs have been reported). ${ }^{125}$

On the other hand, with myometrial, subserosal, or fundal injection, visualization of para-aortic lymphatic drainage increases remarkably, with $16 \%-25 \%$ of para-aortic exclusive SLNs. ${ }^{126}$

In the SENTI-ENDO trial, lymphoscintigraphy and SLN biopsy detected involved nodes in $11 \%$ of cases with a potential difference in 3-year survival of $11 \%$. In the subgroup of patients with low-risk lesions, positive SLNs were the only ones that contained metastases suggesting the therapeutic effect of SLN biopsy. However, the risk of para-aortic lymph node recurrence when micrometastases are detected on pelvic SLN cannot be forgotten, and it justifies the possibility of para-aortic lymphadenectomy. In patients with undetected SLN on lymphoscintigraphy, recurrences were intraoperatively observed in $28.6 \%$ of cases. When SLN was detected, the recurrences diminished to. $12.6 \%$. $^{127}$
Bilateral detection rates are improved when two injection agents (such as blue dye and radiotracer) are used in combination or SPECT/CT is added to planar lymphoscintigraphy. ${ }^{128,129}$

As in cervical cancer, a failed lymphoscintigraphic and intraoperative mapping may lead to a situation in which SLNs are not identified and requires a complete or unilateral lymphadenectomy to exclude disease. ${ }^{130,131}$

Cormier et al proposed the following algorithm: when only unilateral mapping was achieved, a contralateral pelvic lymphadenectomy and en bloc parametrectomy must be performed in all cases. They found $87.5 \%$ patient-based sensitivity and $92.6 \%$ side-specific sensitivity. These figures increased to $100 \%$ when parametrectomy was added and no false-negative results were reported. ${ }^{132}$

Finally, the application of lymphatic mapping for SLN detection must be based on the risk of metastases in the patients. Therefore, in low-risk patients, metastatic involvement of SLN is expected in $1 \%-5 \%$ of cases. The only argument for performing lymphatic mapping in this group might be its upstaging, based on the result of SLN pathologic analysis. ${ }^{125,133-135}$

On the contrary, SLN involvement in the intermediateand high-risk group is high (17.8\% and $21.1 \%$, respectively). These figures warrant the indication for accurate staging, and lymphoscintigraphy plus SLN biopsy may solve this need.

Moreover, there is another theoretical advantage of lymphatic mapping and SLN biopsy: radical lymphadenectomy

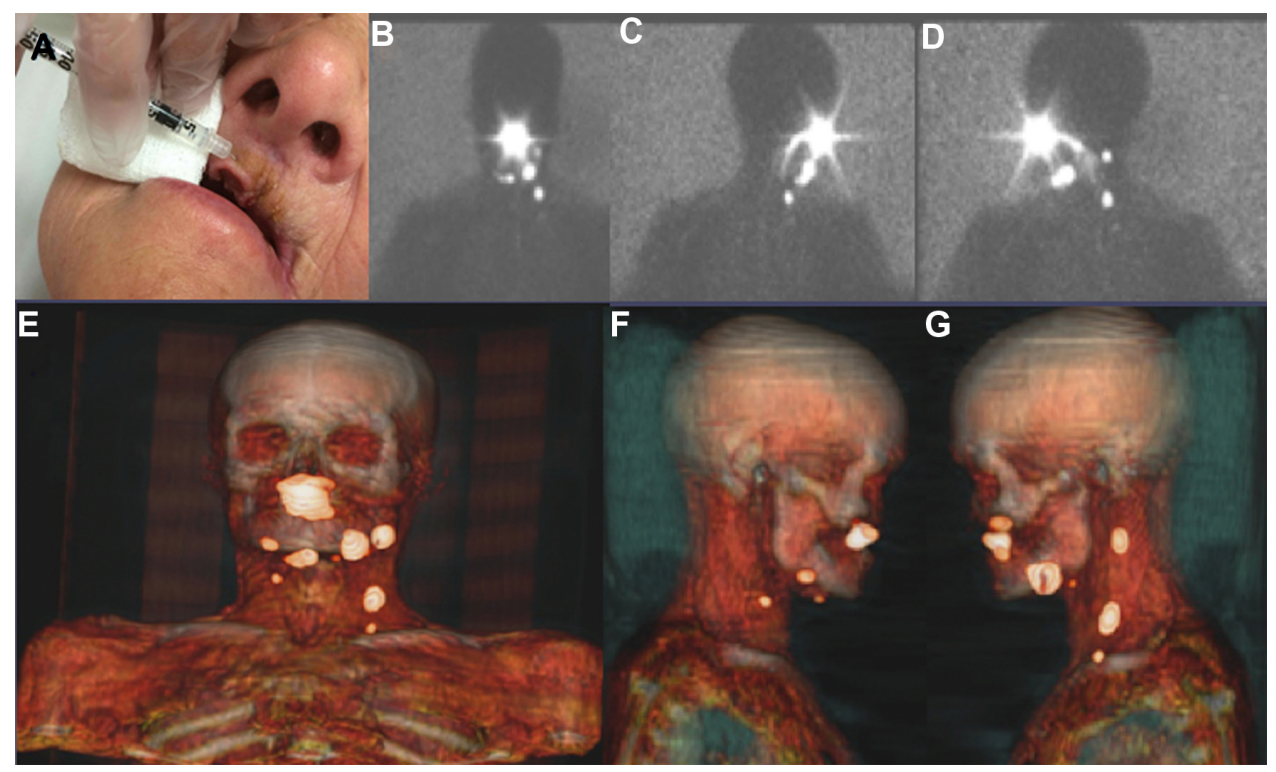

Figure 6 Complex lymphatic drainage assesed in planar and SPECT/CT images.

Notes: Central upper lip squamous cell carcinoma with injection procedure (A). Early planar images in anterior (B), right (C), and left (D) lateral views. The lack of anatomical landscape is improved by the 3D volume rendering reconstruction $(\mathbf{E})-(\mathbf{G})$.

Abbreviation: SPECT/CT, single-photon emission computed tomography/computed tomography. 
could be avoided in nearly $80 \%$ of these latter subgroups of patients. ${ }^{136}$

\section{Oral cavity cancer}

Lymphatic nodes in the head and neck area include 250-300 lymph nodes that are located in various nodal groups. This high lymph node density is a great drawback for the accuracy of the lymphatic mapping and SLN biopsy (Figure 6).

Another important explanation of the drainage variability in this area depends on the location of the primary tumor. For these reasons, lymphoscintigraphy and SPECT/CT are mandatory when lymphatic drainage is explored not only to identify SLNs but also to detect more SLNs in the proximity of the tumors or when aberrant locations exist. Some critical areas for drainage visualization, due to the little distance between the injection site and SLNs, are levels I/II for oral cavity cancer (especially floor-of-mouth) and level II and the parotid area for periauricular melanomas or posterior oral cancers (Figure 7).

SLN biopsy is an alternative method to neck lymphadenectomy in patients with early (T1 or T2) oral cavity carcinoma. This approach may limit the associated morbidity and improve cosmetic outcome. Unfortunately, these lower sensitivity rates are reported for floor-of-mouth cancers as compared with other oral subsites: $80 \%-86 \%$ versus 94\%-97\%, respectively. ${ }^{137,138}$

Currently, the National Comprehensive Cancer Network guidelines stated that sufficient caution must be taken when offering SLN biopsy, particularly in the floor-of-mouth cancer. ${ }^{139}$ The close spatial relation between the injection site and the SLN results in a lower identification rate in lymphoscintigraphy and intraoperative location due to the shine-through effect. SPECT/CT and portable gamma cameras can improve visualization of the relation of SLNs to some important structures, which allows easier, quicker, and safer removal of these nodes. ${ }^{140-142}$

\section{Conclusion}

In conclusion, lymphoscintigraphy is the cornerstone for lymphedema evaluation and lymphatic mapping for SLN biopsy. Consistent use of a chosen technique that includes ${ }^{99 m} \mathrm{mc}-$ labeled tracer and imaging allows a thorough vision of lymphatic vasculature and the depiction of all nodal basins and SLNs. This latter scenario is likely to yield an elevated

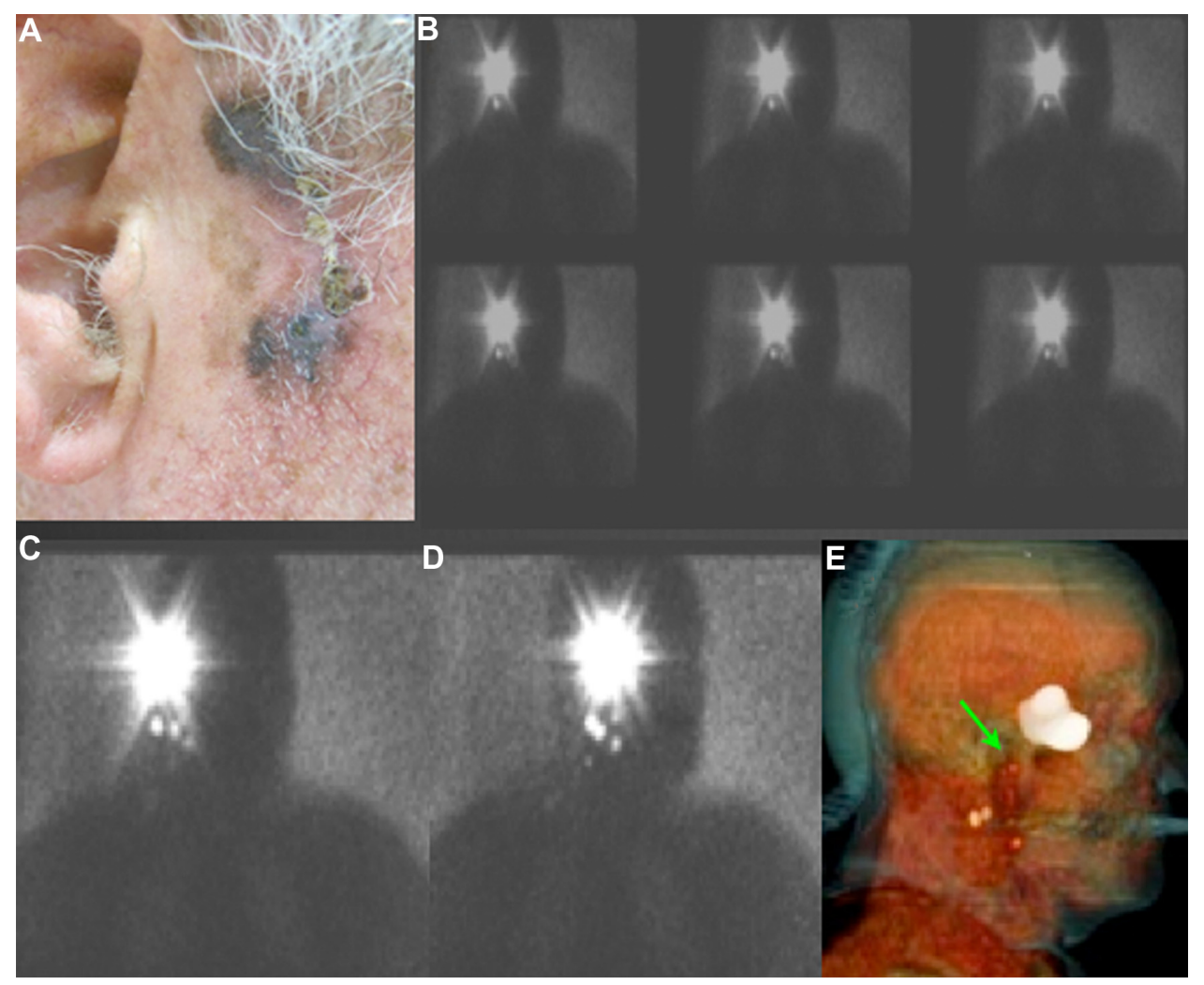

Figure 7 Importance of SPECT/CT in very complex drainage areas (head \& neck).

Notes: Right pre-auricular melanoma (A), dynamic (B), early $(\mathbf{C})$, and delayed (D) images show a very challenging drainage. SPECT/CT reconstructed data (E) allow visualizing a very faint retromandibular node (green arrow), very close to the injection tracer site.

Abbreviation: SPECT/CT, single-photon emission computed tomography/computed tomography. 
identification rate and avoids a significant false-negative rate in breast cancer and other solid tumors. In regions with complex drainage, the addition of SPECT/CT improves the results by detecting additional SLNs in the vicinity of the primary lesions. On the other hand, SPECT/CT is able to provide specific anatomical landmarks to be recognized during the surgical act. Due to this visual information, the paradigm in the SLN approach gradually evolves to "see, open, and recognize."

\section{Acknowledgements}

The authors wish to thank Mrs Marianne Valdés Olmos for her assistance in editing and reviewing the paper.

\section{Disclosure}

The authors report no conflicts of interest in this work.

\section{References}

1. Sherman AI, Ter-Pogossian M. Lymph node concentration of radioactive colloidal gold following interstitial injection. Cancer. 1953;6(6):1238-1240.

2. Hayes S, Janda M, Cornish B, Battistutta D, Newman B. Lymphedema secondary to breast cancer: how choice of measure influences diagnosis, prevalence, and identifiable risk factors. Lymphology. 2008;41(1):18-28.

3. Kramer EL. Lymphoscintigraphy: defining a clinical role. Lymphat Res Biol. 2001;2(1):32-37.

4. Hwang JH, Choi JY, Lee JY, et al. Lymphoscintigraphy predicts response to complex physical therapy in patients with early stage extremity lymphedema. Lymphology. 2007;40(4):172-176.

5. Hellman S. Karnofsky memorial lecture. Natural history of small breast cancers. J Clin Oncol. 1994;12(10):2229-2234.

6. Kazem I, Brady LW, Croll MN, Faust DS, Wolferth CC. The parasternal lymph-node scan as a prognostic test in breast carcinoma. Radiology. 1969;92(3):617-620.

7. Xiong L, Engel H, Gazyakan E, et al. Current techniques for lymphatic imaging: state of the art and future perspectives. Eur J Surg Oncol. 2014;40(3):270-276.

8. Warren AG, Brorson H, Borud LJ, Slavin SA. Lymphedema: a comprehensive review. Ann Plast Surg. 2007;59(4):464-472.

9. Schook CC, Mulliken JB, Fishman SJ, Grant FD, Zurakowski D, Greene AK. Primary lymphedema: clinical features and management in 138 pediatric patients. Plast Reconstr Surg. 2011;127(6):2419-2431.

10. Ozaslan C, Kuru B. Lymphedema after treatment of breast cancer. $\mathrm{Am}$ J Surg. 2004;187(1):69-72.

11. Hinrichs CS, Watroba NL, Rezaishiraz H, et al. Lymphedema secondary to postmastectomy radiation: incidence and risk factors. Ann Surg Oncol. 2004;11(6):573-580.

12. Ohba Y, Todo Y, Kobayashi N, et al. Risk factors for lower-limb lymphedema after surgery for cervical cancer. Int J Clin Oncol. 2011;16(3):238-243.

13. Tiwari A, Cheng KS, Button M, Myint F, Hamilton G. Differential diagnosis, investigation, and current treatment of lower limb lymphedema. Arch Surg. 2003;138(2):152-161.

14. Stanton AW, Modi S, Mellor RH, Levick JR, Mortimer PS. Recent advances in breast cancer-related lymphedema of the arm: lymphatic pump failure and predisposing factors. Lymphat Res Biol. 2009;7(1):29-45.

15. McLaughlin SA. Lymphedema: separating fact from fiction. Oncology. 2012;26(3):242-249.

16. Wojcinski S, Nuengsrl S, Hillemanns P, et al. Axillary dissection in primary breast cancer: variations of the surgical technique and influence on morbidity. Cancer Manag Res. 2012;4:121-127.
17. Szuba A, Shin WS, Strauss HW, Rockson S. The third circulation: radionuclide lymphoscintigraphy in the evaluation of lymphedema. J Nucl Med. 2003;44(1):43-57.

18. Williams WH, Witte CL, Witte MH, McNeill GC. Radionuclide lymphangioscintigraphy in the evaluation of peripheral lymphedema. Clin Nucl Med. 2000;25(6):451-464.

19. Szuba A, Strauss W, Sirsikar SP, Rockson SG. Quantitative radionuclide lymphoscintigraphy predicts outcome of manual lymphatic therapy in breast cancer-related lymphedema of the upper extremity. Nucl Med Commun. 2002;23(12):1171-1175.

20. Kim YB, Hwang JH, Kim TW, Chang HJ, Lee SG. Would complex decongestive therapy reveal long term effect and lymphoscintigraphy predict the outcome of lower-limb lymphedema related to gynecologic cancer treatment? Gynecol Oncol. 2012;127(3):638-642.

21. Pecking AP, Albérini JL, Wartski M, Edeline V, Cluzan RV. Relationship between lymphoscintigraphy and clinical findings in lower limb lymphedema (LO): toward a comprehensive staging. Lymphology. 2008;41(1):1-10.

22. Giammarile F, Alazraki N, Aarsvold JN, et al. The EANM and SNMMI practice guideline for lymphoscintigraphy and sentinel node localization in breast cancer. Eur J Nucl Med Mol Imaging. 2013;40(12): 1932-1947.

23. Hung JC, Wiseman GA, Wahner HW, Mullan BP, Taggart TR, Dunn WL. Filtered technetium-99m-sulfur colloid evaluated for lymphoscintigraphy. J Nucl Med. 1995;36(10):1895-1901.

24. Mostbeck A, Partsch H. Isotope lymphography: possibilities and limits in evaluation of lymph transport. Wien Med Wochenschr. 1999;149(2-4):87-91.

25. Partsch $\mathrm{H}$. Assessment of abnormal lymph drainage for the diagnosis of lymphedema by isotopic lymphangiography and by indirect lymphography. Clin Dermatol. 1995;13(5):445-450.

26. Erba PA, Solllini M, D’Errico G, Mariani G. Methodological aspects of lymphoscintigraphy: bicompartimental versus monocompartimental radiocolloid administration. In: Mariani G, Manca G, Valdés AR, Orsini F, Vidal-Sicart S, editors. Atlas of Lymphoscintigraphy and Sentinel Node Mapping. A Pictorial Case-Based Approach. Milan: Springer-Verlag Milan; 2013:27-38.

27. Bräutigam P, Földi E, Schaiper I, Krause T, Vanscheidt W, Moser E. Analysis of lymphatic drainage in various forms of leg edema using two compartment lymphoscintigraphy. Lymphology. 1998;31(2): $43-55$.

28. Karaçavuş S, Yılmaz YK, Ekim H. Clinical significance of lymphoscintigraphy findings in the evaluation of lower extremity lymphedema. Mol Imaging Radionucl Ther. 2015;24(2):80-84.

29. Moshiri M, Katz DS, Boris M, Yung E. Using lymphoscintigraphy to evaluate suspected lymphedema of the extremities. AJR Am J Roentgenol. 2002;178(2):405-412.

30. Infante JR, García L, Laguna P, et al. Lymphoscintigraphy for differential diagnosis of peripheral edema: diagnostic yield of different scintigraphic patterns. Rev Esp Med Nucl Imagen Mol. 2012;31(5):237-242.

31. Bourgeois P, Leduc O, Belgrado JP, Leduc A. Scintigraphic investigations of the superficial lymphatic system: quantitative differences between intradermal and subcutaneous injections. Nucl Med Commun. 2009;30(4):270-274.

32. Tartaglione G, Pagan M, Morese R, et al. Intradermal lymphoscintigraphy at rest and after exercise: a new technique for the functional assessment of the lymphatic system in patients with lymphoedema. Nucl Med Commun. 2010;31(6):547-551.

33. Carena M, Campini R, Zelaschi G, Rossi G, Aprile C, Paroni G. Quantitative lymphoscintigraphy. Eur J Nucl Med. 1988;14(2): $88-92$.

34. Dabrowski J, Merkert R, Kuśmierek J. Optimized lymphoscintigraphy and diagnostics of lymphatic oedema of the lower extremities. Nucl Med Rev Cent East Eur. 2008;11(1):26-29.

35. Weissleder H, Weissleder R. Lymphedema: evaluation of qualitative and quantitative lymphoscintigraphy in 238 patients. Radiology. 1988;167(3):729-735. 
36. Scarsbrook AF, Ganeshan A, Bradley KM. Pearls and pitfalls of radionuclide imaging of the lymphatic system part 2: evaluation of extremity lymphoedema. Br J Radiol. 2007;80(951):219-226.

37. Stanton AW, Modi S, Mellor RH, et al. A quantitative lymphoscintigraphic evaluation of lymphatic function in the swollen hands of women with lymphoedema following breast cancer treatment. Clin Sci (Lond). 2006;110(5):553-561.

38. Gironet N, Baulieu F, Giraudeau B, et al. [Lymphedema of the limb: predictors of efficacy of combined physical therapy]. Ann Dermatol Venereol. 2004;131(8-9):775-779.

39. Slavin SA, Upton J, Kaplan WD, Van den Abbeele AD. An investigation of lymphatic function following free-tissue transfer. Plast Reconstr Surg 1997;99(3):730-741.

40. Weiss M, Baumeister RG, Hahn K. Planning and monitoring of autologous lymph vessel transplantation by means of nuclear medicine lymphoscintigraphy. Handchir Mikrochir Plast Chir. 2003;35(4): 210-215.

41. Frühling JG, Bourgeois P. Axillary lymphoscintigraphy: current status in the treatment of breast cancer. Crit Rev Oncol Hematol. 1983;1(1):1-20.

42. Das IJ, Cheville AL, Scheuermann J, Srinivas SM, Alavi A, Solin LJ. Use of lymphoscintigraphy in radiation treatment of primary breast cancer in the context of lymphedema risk reduction. Radiother Oncol. 2011;100(2):293-298.

43. Cabanas RM. An approach for the treatment of penile carcinoma. Cancer. 1977;39(2):456-466.

44. Morton DL, Wen DR, Wong JH, et al. Technical details of intraoperative lymphatic mapping for early stage melanoma. Arch Surg 1992;127(4):392-399.

45. Gershenwald JE, Thompson W, Mansfield PF, et al. Multi-institutional melanoma lymphatic mapping experience: the prognostic value of sentinel lymph node status in 612 stage I or II melanoma patients. J Clin Oncol. 1999;17(3):976-983.

46. McMasters KM, Tuttle TM, Carlson DJ, et al. Sentinel lymph node biopsy for breast cancer: a suitable alternative to routine axillary dissection in multi-institutional practice when optimal technique is used. J Clin Oncol. 2000;18(13):2560-2566.

47. Kitajima M, Kitagawa Y. Universal applications of sentinel node technology. Ann Surg Oncol. 2004;11(3 Suppl):144S-146S.

48. Nieweg OE, Uren RF, Thompson JF. The history of sentinel lymph node biopsy. Cancer J. 2015;21(1):3-6.

49. Uren RF, Howman-Giles RB, Shaw HM, Thompson JF, McCarthy WH. Lymphoscintigraphy in high-risk melanoma of the trunk: predicting draining node groups, defining lymphatic channels and locating the sentinel node. J Nucl Med. 1993;34(9):1435-1440.

50. O'Brien CJ, Uren RF, Thompson JF, et al. Prediction of potential metastatic sites in cutaneous head and neck melanoma using lymphoscintigraphy. Am J Surg. 1995;170(5):461-466.

51. Kamath D, Rapaport D, DeConti R, et al. Redefining cutaneous lymphatic flow: the necessity of preoperative lymphoscintigraphy in the management of malignant melanoma. J Fla Med Assoc. 1997;84(3): 182-187.

52. Valdés Olmos RA, Hoefnagel CA, Nieweg OE, et al. Lymphoscintigraphy in oncology: a rediscovered challenge. Eur J Nucl Med. 1999 26(4 Suppl):S2-S10.

53. Alazraki NP, Styblo T, Grant SF, Cohen C, Larsen T, Aarsvold JN. Sentinel node staging of early breast cancer using lymphoscintigraphy and the intraoperative gamma-detecting probe. Semin Nucl Med. 2000;30(1):56-64.

54. Paganelli G, De Cicco C, Chinol M. Sentinel node localization by lymphoscintigraphy: a reliable technique with widespread applications. Recent Results Cancer Res. 2000;157:121-129.

55. Bluemel C, Herrmann K, Giammarile F, et al. EANM practice guidelines for lymphoscintigraphy and sentinel lymph node biopsy in melanoma. Eur J Nucl Med Mol Imaging. 2015;42(11):1750-1766.

56. Moncayo VM, Aarsvold JN, Alazraki NP. Lymphoscintigraphy and sentinel nodes. J Nucl Med. 2015;56(6):901-907.
57. Taylor A Jr, Murray D, Herda S, Vansant J, Alazraki N. Dynamic lymphoscintigraphy to identify the sentinel and satellite nodes. Clin $\mathrm{Nucl}$ Med. 1996;21(10):755-758.

58. Uren RF, Howman-Giles RB, Thompson JF. Variation in cutaneous lymphatic flow rates. Ann Surg Oncol. 1997;4(3):279-280.

59. Kapteijn BA, Nieweg OE, Valdés Olmos RA, et al. Reproducibility of lymphoscintigraphy for lymphatic mapping in cutaneous melanoma. J Nucl Med. 1996;37(6):972-975.

60. Tonakie A, Sondak V, Yahanda A, Wahl RL. Reproducibility of lymphoscintigraphic drainage patterns in sequential 99mTc human serum albumin and $99 \mathrm{mTc}$ sulfur colloid studies: implications for sentinel node identification in melanoma. Surgery. 1999;126(5):955-962.

61. Rettenbacher L, Koller J, Kässmann H, Holzmannhofer J, Rettenbacher T, Galvan G. Reproducibility of lymphoscintigraphy in cutaneous melanoma: can we accurately detect the sentinel lymph node by expanding the tracer injection distance from the tumor site? J Nucl Med. 2001;42(3):424-429.

62. Uren RF, Howman-Giles R, Chung DK, Morton RL, Thompson JF. The reproducibility in routine clinical practice of sentinel lymph node identification by pre-operative lymphoscintigraphy in patients with cutaneous melanoma. Ann Surg Oncol. 2007;14(2):899-905.

63. Vidal M, Vidal-Sicart S, Torrents A, et al. Accuracy and reproducibility of lymphoscintigraphy for sentinel node detection in patients with cutaneous melanoma. J Nucl Med. 2012;53(8):1193-1199.

64. Vitali GC, Trifirò G, Zonta M, et al. Lymphoscintigraphy in clinical routine practice: reproducibility and accuracy in melanoma patients with a long-term follow-up. Eur J Surg Oncol. 2014;40(1):55-60.

65. Tanis PJ, Valdés Olmos RA, Muller SH, Nieweg OE. Lymphatic mapping in patients with breast carcinoma: reproducibility of lymphoscintigraphic results. Radiology. 2003;228(2):546-551.

66. Kroon BK, Valdés Olmos RA, van Tinteren H, Nieweg OE, Horenblas S. Reproducibility of lymphoscintigraphy for lymphatic mapping in patients with penile carcinoma. J Urol. 2005;174(6):2214-2217.

67. Rubello D, Zavagno G, Bozza F, et al. Analysis of technical and clinical variables affecting sentinel node localization in patients with breast cancer after a single intradermal injection of $99 \mathrm{mTc}$ nanocolloidal albumin. Nucl Med Commun. 2004;25(11):1119-1124.

68. Alazraki N, Glass EC, Castronovo F, Olmos RA, Podoloff D; Society of Nuclear Medicine. Procedure guideline for lymphoscintigraphy and the use of intraoperative gamma probe for sentinel lymph node localization in melanoma of intermediate thickness 1.0. $\mathrm{J} \mathrm{Nucl} \mathrm{Med}$. 2002;43(10):1414-1418.

69. Mariani G, Erba P, Manca G, et al. Radioguided sentinel lymph node biopsy in patients with malignant cutaneous melanoma: the nuclear medicine contribution. J Surg Oncol. 2004;85(3):141-151.

70. Mariani G, Erba P, Villa G, et al. Lymphoscintigraphic and intraoperative detection of the sentinel lymph node in breast cancer patients: the nuclear medicine perspective. J Surg Oncol. 2004;85(3):112-122.

71. Valdés Olmos RA, Rietbergen DD, Vidal-Sicart S, Manca G, Giammarile F, Mariani G. Contribution of SPECT/CT imaging to radioguided sentinel lymph node biopsy in breast cancer, melanoma, and other solid cancers: from "open and see" to "see and open". Q J Nucl Med Mol Imaging. 2014;58(2):127-139.

72. Collarino A, Donswijk ML, van Driel WJ, Stokkel MP, Valdés Olmos RA. The use of SPECT/CT for anatomical mapping of lymphatic drainage in vulvar cancer: possible implications for the extent of inguinal lymph node dissection. Eur J Nucl Med Mol Imaging. 2015;42(13):2064-2071.

73. Chícharo de Freitas JR, KleinJan GH, van der Poel HG, et al. Utility of SPECT/CT scan for anatomical localization of pararectal and presacral sentinel nodes in prostate cancer. Rev Esp Med Nucl Imagen Mol. 2015;34(1):19-23.

74. Vidal-Sicart S, Brouwer OR, Valdés-Olmos RA. Evaluation of the sentinel lymph node combining SPECT/CT with the planar image and its importance for the surgical act. Rev Esp Med Nucl. 2011;30(5): $331-337$.

75. Vermeeren L, Valdés Olmos RA, Klop WM, BalmAJ, van den Brekel MW. A portable gamma-camera for intraoperative detection of sentinel nodes in the head and neck region. J Nucl Med. 2010;51(5):700-703. 
76. Zaknun JJ, Giammarile F, Olmos RA, Vidal-Sicart S, Mariani G. Changing paradigms in radioguided surgery and intraoperative imaging: the GOSTT concept. Eur J Nucl Med Mol Imaging. 2012;39(1):1-3.

77. Van Den Berg NS, Buckle T, Kleinjan GI, et al. Hybrid tracers for sentinel node biopsy. Q J Nucl Med Mol Imaging. 2014;58(2):193-206.

78. Frontado LM, Brouwer OR, van den Berg NS, et al. Added value of the hybrid tracer indocyanine green-99mTc-nanocolloid for sentinel node biopsy in a series of patients with different lymphatic drainage patterns. Rev Esp Med Nucl Imagen Mol. 2013;32(4):227-233.

79. Vidal-Sicart S, Rioja ME, Paredes P, Keshtgar MR, Valdés Olmos RA. Contribution of perioperative imaging to radioguided surgery. $Q \mathrm{~J} \mathrm{Nucl}$ Med Mol Imaging. 2014;58(2):140-160.

80. Qin Z, Hall DJ, Liss MA, et al. Optimization via specific fluorescence brightness of a receptor-targeted probe for optical imaging and positron emission tomography of sentinel lymph nodes. J Biomed Opt. 2013;18(10):101315.

81. Qin Z, Hoh CK, Hall DJ, Vera DR. A tri-modal molecular imaging agent for sentinel lymph node mapping. Nucl Med Biol. 2015;42(12):917-922.

82. Liss MA, Farshchi-Heydari S, Qin Z, et al. Preclinical evaluation of robotic-assisted sentinel lymph node fluorescence imaging. J Nucl Med. 2014;55(9):1552-1556.

83. Liss MA, Stroup SP, Qin Z, et al. Robotic-assisted fluorescence sentinel lymph node mapping using multimodal image guidance in an animal model. Urology. 2014;84(4):982e9-982e14.

84. Lin D, Franc BL, Kashani-Sabet M, Singer MI. Lymphatic drainage patterns of the head and neck: cutaneous melanoma observed on lymphoscintigraphy and sentinel lymph node biopsy. Head Neck. 2006;28(3):249-255.

85. Parrett BM, Kashani-Sabet M, Singer M, et al. Long-term prognosis and significance of the sentinel lymph node in head and neck melanoma. Otolaryngol Head Neck Surg. 2012;147(4):699-706.

86. Roy JM, Whitfield RJ, Gill PG. Review of the role of sentinel node biopsy in cutaneous head and neck melanoma. ANZ J Surg. Epub 2015 Sep 11.

87. Kretschmer L, Bertsch HP, Zapf A, et al. Nodal basin recurrence after sentinel lymph node biopsy for melanoma: a retrospective multicenter study in 2653 patients. Medicine (Baltimore). 2015;94(36):e1433.

88. Ho AM, Avery R, Krupinski EA, Warneke J, Kuo PH. Predictive role of imaging in sentinel lymph node dissection for melanoma. Lymphology. 2014;47(3):134-141.

89. Luo S, Lobo AZ, Tanabe KK, et al. Clinical significance of microscopic melanoma metastases in the nonhottest sentinel lymph nodes. JAMA Surg. 2015;150(5):465-472.

90. Bennie G, Vorster M, Buscombe J, Sathekge M. The added value of a single-photon emission computed tomography-computed tomography in sentinel lymph node mapping in patients with breast cancer and malignant melanoma. World J Nucl Med. 2015;14(1):41-46.

91. Stoffels I, Boy C, Pöppel T, et al. Association between sentinel lymph node excision with or without preoperative SPECT/CT and metastatic node detection and disease-free survival in melanoma. JAMA. 2012;308(10):1007-1014

92. Veenstra HJ, Vermeeren L, Olmos RA, Nieweg OE. The additional value of lymphatic mapping with routine SPECT/CT in unselected patients with clinically localized melanoma. Ann Surg Oncol. 2012;19(3):1018-1023.

93. Goyal A, Newcombe RG, Mansel RE, et al. Role of routine preoperative lymphoscintigraphy in sentinel node biopsy for breast cancer. Eur J Cancer. 2005;41(2):238-243.

94. Uren RF, Howman-Giles R, Renwick SB, Gillett D. Lymphatic mapping of the breast: locating the sentinel lymph nodes. World J Surg. 2001;25(6):789-793.

95. Hindié E, Groheux D, Brenot-Rossi I, Rubello D, Moretti JL, Espié M. The sentinel node procedure in breast cancer: nuclear medicine as the starting point. J Nucl Med. 2011;52(3):405-414.

96. Moncayo VM, Aarsvold JN, Grant SF, Bartley SC, Alazraki NP. Status of sentinel lymph node for breast cancer. Semin Nucl Med. 2013;43(4):281-293.
97. Estourgie SH, Nieweg OE, Olmos RA, Rutgers EJ, Kroon BB. Lymphatic drainage patterns from the breast. Ann Surg. 2004;239(2): 232-237.

98. Huang O, Wang L, Shen K, et al. Breast cancer subpopulation with high risk of internal mammary lymph nodes metastasis: analysis of 2,269 Chinese breast cancer patients treated with extended radical mastectomy. Breast Cancer Res Treat. 2008;107(3):379-387.

99. Yao MS, Kurland BF, Smith AH, et al. Internal mammary nodal chain drainage is a prognostic indicator in axillary node-positive breast cancer. Ann Surg Oncol. 2007;14(10):2985-2993.

100. Colleoni M, Zahrieh D, Gelber RD, et al. Site of primary tumor has a prognostic role in operable breast cancer: International Breast Cancer Study Group experience. J Clin Oncol. 2005;23(7):1390-1400.

101. Chen RC, Lin NU, Golshan M, Harris JR, Bellon JR. Internal mammary nodes in breast cancer: diagnosis and implications for patient management. A systematic review. J Clin Oncol. 2008;26(30):4981-4989.

102. Veronesi U, Arnone P, Veronesi P, et al. The value of radiotherapy on metastatic internal mammary nodes in breast cancer. Results on a large series. Ann Oncol. 2008;19(9):1553-1560.

103. Heuts EM, van der Ent FW, von Meyenfeldt MF, Voogd AC. Internal mammary lymph drainage and sentinel node biopsy in breast cancer - a study on 1008 patients. Eur J Surg Oncol. 2009;35(3):252-257.

104. Hindié E, Groheux D, Hennequin C, et al. Lymphoscintigraphy can select breast cancer patients for internal mammary chain radiotherapy. Int J Radiat Oncol Biol Phys. 2012;83(4):1081-1088.

105. Vermeeren L, Valdés Olmos RA, Meinhardt W, et al. Value of SPECT/CT for detection and anatomic localization of sentinel lymph nodes before laparoscopic sentinel node lymphadenectomy in prostate cancer. J Nucl Med. 2009;50(6):865-870.

106. Meinhardt W, van der Poel HG, Valdés Olmos RA, Bex A, Brouwer OR, Horenblas S. Laparoscopic sentinel lymph node biopsy for prostate cancer: the relevance of locations outside the ex-tended dissection area. Prostate Cancer. 2012;2012:751753.

107. Vermeeren L, Meinhardt W, van der Poel HG, Valdés Olmos RA. Lymphatic drainage from the treated versus untreated prostate: feasibility of sentinel node biopsy in recurrent cancer. Eur J Nucl Med Mol Imaging. 2010;37(11):2012-2026.

108. de Bonilla-Damiá A, Roberto Brouwer O, Meinhardt W, ValdésOlmos RA. Lymphatic drainage in prostate carcinoma assessed by lymphoscintigraphy and SPECT/CT: its importance for the sentinel node procedure. Rev Esp Med Nucl Imagen Mol. 2012;31(2):66-70.

109. Valdés Olmos RA, Tanis PJ, Hoefnagel CA, et al. Penile lymphoscintigraphy for sentinel node identification. Eur J Nucl Med. 2001;28(5): 581-585.

110. Leijte JA, Valdés Olmos RA, Nieweg OE, Horenblas S. Anatomical mapping of lymphatic drainage in penile carcinoma with SPECT-CT: implications for the extent of inguinal lymph node dissection. Eur Urol. 2008;54(4):885-890.

111. Paño B, Sebastià C, Ripoll E, et al. Pathways of lymphatic spread in gynecologic malignancies. Radiographics. 2015;35(3):916-945.

112. Lindell G, Jonsson C, Ehrsson RJ, et al. Evaluation of preoperative lymphoscintigraphy and sentinel node procedure in vulvar cancer. Eur J Obstet Gynecol Reprod Biol. 2010;152(1):91-95.

113. Martínez-Palones JM, Pérez-Benavente MA, Gil-Moreno A, et al. Comparison of recurrence after vulvectomy and lymphadenectomy with and without sentinel node biopsy in early stage vulvar cancer. Gynecol Oncol. 2006;103(3):865-870.

114. Klapdor R, Länger F, Gratz KF, Hillemanns P, Hertel H. SPECT/CT for SLN dissection in vulvar cancer: improved SLN detection and dissection by preoperative three-dimensional anatomical localisation. Gynecol Oncol. 2015;138(3):590-596.

115. Levenback CF, Ali S, Coleman RL, et al. Lymphatic mapping and sentinel lymph node biopsy in women with squamous cell carcinoma of the vulva: a gynecologic oncology group study. J Clin Oncol. 2012;30(31):3786-3791.

116. Van der Zee AG, Oonk MH, De Hulla JA, et al. Sentinel node dissection is safe in the treatment of early-stage vulvar cancer. J Clin Oncol. 2008;26(6):884-889. 
117. De Hullu JA, van der Zee AG. Surgery and radiotherapy in vulvar cancer. Crit Rev Oncol Hematol. 2006;60(1):38-58.

118. Woelber L, Kock L, Gieseking F, et al. Clinical management of primary vulvar cancer. Eur J Cancer. 2011;47(15):2315-2321.

119. Coleman RL, Ali S, Levenback CF, et al. Is bilateral lymphadenectomy for midline squamous carcinoma of the vulva always necessary? An analysis from Gynecologic Oncology Group (GOG)173. Gynecol Oncol. 2013;128(2):155-159.

120. Lécuru F, Mathevet P, Querleu D, et al. Bilateral negative sentinel nodes accurately predict absence of lymph node metastasis in early cervical cancer: results of the SENTICOL study. J Clin Oncol. 2011;29(13): 1686-1691.

121. Bats AS, Frati A, Mathevet P, et al. Contribution of lymphoscintigraphy to intraoperative sentinel lymph node detection in early cervical cancer: analysis of the prospective multicenter SENTICOL cohort. Gynecol Oncol. 2015;137(2):264-269.

122. Klapdor R, Mücke J, Schneider M, et al. Value and advantages of preoperative sentinel lymph node imaging with SPECT/CT in cervical cancer. Int J Gynecol Cancer. 2014;24(2):295-302.

123. Hoogendam JP, Veldhuis WB, Hobbelink MG, Verheijen RH, van den Bosch MA, Zweemer RP. 99mTc SPECT/CT versus planar lymphoscintigraphy for preoperative sentinel lymph node detection in cervical cancer: a systematic review and metaanalysis. J Nucl Med. 2015;56(5):675-680.

124. Giammarile F, Bozkurt MF, Cibula D, et al. The EANM clinical and technical guidelines for lymphoscintigraphy and sentinel node localization in gynaecological cancers. Eur J Nucl Med Mol Imaging. 2014;41(7):1463-1477.

125. Ballester M, Dubernard G, Lécuru F, et al. Detection rate and diagnostic accuracy of sentinel-node biopsy in early stage endometrial cancer: a prospective multicentre study (SENTI-ENDO). Lancet Oncol. 2011;12(5):469-476.

126. Frumovitz M, Bodurka DC, Broaddus RR, et al. Lymphatic mapping and sentinel node biopsy in women with high-risk endometrial cancer. Gynecol Oncol. 2007;104(1):100-103.

127. Daraï E, Dubernard G, Bats AS, et al. Sentinel node biopsy for the management of early stage endometrial cancer: long-term results of the SENTI-ENDO study. Gynecol Oncol. 2015;136(1):54-59.

128. Sawicki S, Kobierski J, Lass P, Cytawa W, Wydra D. Preoperative detection of sentinel lymph nodes in endometrial cancer using SPECT/CT. Clin Nucl Med. 2013;38(9):726-729.

129. Naaman Y, Pinkas L, Roitman S, et al. The added value of SPECT/CT in sentinel lymph nodes mapping for endometrial carcinoma. Ann Surg Oncol. 2016;23(2):450-455.
130. Abu-Rustum NR. Sentinel lymph node mapping for endometrial cancer: a modern approach to surgical staging. J Natl Compr Canc Netw. 2014;12(2):288-297.

131. Morice P, Leary A, Creutzberg C, Abu-Rustum N, Darai E. Endometrial cancer. Lancet. Epub 2015 Sep 4.

132. Cormier B, Rozenholc AT, Gotlieb W, et al; Communities of Practice (CoP) Group of Society of Gynecologic Oncology of Canada (GOC). Sentinel lymph node procedure in endometrial cancer: a systematic review and proposal for standardization of future research. Gynecol Oncol. 2015;138(2):478-485.

133. Sawicki S, Lass P, Wydra D. Sentinel lymph node biopsy in endometrial cancer. Comparison of 2 detection methods. Int J Gynecol Cancer. 2015;25(6):1044-1050.

134. Niikura H, Kaiho Sakuma M, Tokunaga H, et al. Tracer injection sites and combinations for sentinel lymph node detection in patients with endometrial cancer. Gynecol Oncol. 2013;131(2):299-303.

135. Raimond E, Ballester M, Hudry D, et al. Impact of sentinel lymph node biopsy on the therapeutic management of early-stage endometrial cancer: results of a retrospective multicenter study. Gynecol Oncol. 2014;133(3):506-511.

136. Cibula D, Oonk MH, Abu-Rustum NR. Sentinel lymph node biopsy in the management of gynecologic cancer. Curr Opin Obstet Gynecol. 2015;27(1):66-72.

137. Alkureishi LW, Ross GL, Shoaib T, et al. Sentinel node biopsy in head and neck squamous cell cancer: 5-year follow-up of a European multicentre trial. Ann Surg Oncol. 2010;17(9):2459-2464.

138. Pedersen NJ, Jensen HD, Hedbäck N, et al. Staging of early lymph node metastases with the sentinel lymph node technique and predictive factors in T1/T2 oral cavity cancer: a retrospective single-center study of 253 consecutive patients. Head Neck. Epub 2015 Jun 3.

139. National Comprehensive Cancer Network [homepage on the Internet]. Available from: www.nccn.org. Accessed October 3, 2015.

140. Haerle SK, Hany TF, Strobel K, Sidler D, Stoeckli SJ. Is there an additional value of SPECT/CT over planar lymphoscintigraphy for sentinel node mapping in oral/oropharyngeal squamous cell carcinoma? Ann Surg Oncol. 2009;16(11):3118-3124.

141. Mayoral M, Paredes P, Sieira R, Vidal-Sicart S, Marti C, Pons F. The added value of a portable gamma camera for intraoperative detection of sentinel lymph node in squamous cell carcinoma of the oral cavity: a case report. Rev Esp Med Nucl Imagen Mol. 2014;33(4):237-240.

142. Hellingman D, de Wit-van der Veen LJ, Klop WM, Olmos RA. Detecting near-the-injection-site sentinel nodes in head and neck melanomas with a high-resolution portable gamma camera. Clin Nucl Med. 2015;40(1):e11-e16.
Research and Reports in Nuclear Medicine

\section{Publish your work in this journal}

Research and Reports in Nuclear Medicine is an international, peerreviewed, open access journal publishing original research, reports, reviews and commentaries on all areas of nuclear medicine. The manuscript management system is completely online and includes a very

\section{Dovepress}

quick and fair peer-review system. Visit http://www.dovepress.com/ testimonials.php to read real quotes from published authors. 